\title{
Análisis de las prestaciones de los sistemas LTE y LTE-Advanced a partir de procesos de simulación
}

\author{
Analysis of LTE and LTE-Advanced system features through simulation processes
}

\author{
Giselle González ${ }^{1 *} \quad$ Danaisy Prado ${ }^{2} \quad$ Francisco Marante ${ }^{3} \quad$ Arielys Ledesma $^{4}$
}

Recibido 20 de octubre de 2015, aceptado 16 de septiembre de 2016

Received: October 20, 2015 Accepted: September 16, 2016

\begin{abstract}
RESUMEN
Hoy en día el desarrollo vertiginoso de las comunicaciones móviles, promovido por la cada vez más creciente demanda de tráfico, impulsa el despliegue de redes con mejores throughputs y menores latencias. En esta investigación se hace una comparación de los sistemas LTE y LTE-Advanced por medio de un estudio de los parámetros que a nivel de enlace influyen en la tasa de datos que es experimentada por el usuario. Para ello, se compara el desempeño del enlace de comunicación entre un usuario y su estación base, empleando los simuladores LTE y LTE-Advanced Link Level desarrollados por la Universidad Tecnológica de Viena. Los resultados obtenidos muestran las ventajas de LTE-Advanced respecto a LTE, dadas por el empleo de modos de transmisión superiores. De esta forma se destaca la capacidad de las técnicas MIMO para aprovechar las características intrínsecas del canal de transmisión.
\end{abstract}

Palabras clave: LTE, Throughput, MIMO, estación base, equipo de usuario, subtrama, canal de radio.

\begin{abstract}
Nowadays' ongoing development of mobile communications, promoted by the dramatically risen in traffic demand, impulses the deployment of networks with higher throughputs and lower latencies. In this paper, a comparison is made between LTE and LTE-Advanced systems by conducting a study about the parameters, wich at a link level influence the most the data rate experienced by the user. To do so, the LTE and LTE-Advanced Downlink Link Level Simulators developed by the Universidad Tecnológica de Vienna are employed to analyze the communication link between a single user and its Base Station. The results obtained allow to appreciate LTE-Advanced advantages when compared to LTE, given by the usage of superior transmitting modes. Thus, the capacity of MIMO techniques to exploit the intrinsic characteristics of the transmission channel is emphasized.
\end{abstract}

Keywords: LTE, Throughput, MIMO, base station, user equipment, sub frame, radio channel.

1 Departamento de Comunicaciones Inalámbricas. Instituto Superior Politécnico José Antonio Echeverría. Calle 14 № 11901 , Marianao. La Habana, Cuba. E-mail: giselle2207@ gmail.com

2 Tecnología de la Información. MOVITEL. Avenida 49 № 2831, Reparto Kohly, Playa. La Habana, Cuba. E-mail: dpradoalvarez@gmail.com

3 Departamento de Comunicaciones Inalámbricas. Instituto Superior Politécnico José Antonio Echeverría. Calle 14 N 11901 , Marianao. La Habana, Cuba. E-mail: marante@electrica.cujae.edu.cu

4 Departamento de Operaciones de la Red. ETECSA. Calle Maceo No 108, San Cristobal, Artemisa. La Habana, Cuba. E-mail: arielys.ledesma@etecsa.cu

* Autor de correspondencia 


\section{INTRODUCCIÓN}

\section{Antecedentes generales}

Los sistemas de cuarta generación constituyen una de las más novedosas tecnologías móviles desplegadas hasta hoy. A pesar de que se han estudiado ya por varios años, y son bien elevadas las prestaciones alcanzadas a nivel de laboratorio, en muy pocos lugares se ha logrado un total despliegue de todas sus funcionalidades, y no se encuentran por tanto operando en la totalidad de su capacidad. Resulta entonces sumamente importante la realización de un estudio del desempeño de los sistemas LTE y LTE-Advanced ante una variedad de escenarios de simulación, para determinar bajo cuáles circunstancias se produce su mejor y su peor desempeño.

\section{Cuarta generación (4G)}

4G son las siglas de la cuarta generación de los sistemas de comunicaciones móviles y están basados totalmente en el protocolo de transmisión IP, siendo considerados un sistema de sistemas y una red de redes. $4 \mathrm{G}$ se utiliza en un sentido amplio para definir varios tipos de acceso móvil de banda ancha, no solo sistemas de telefonía celular, tanto en interiores (indoor) como en exteriores (outdoor), con alta QoS y óptima seguridad [1], permitiendo la oferta de servicios de cualquier clase en cualquier momento y en cualquier lugar, con el mínimo costo posible [2].

LTE-Advanced es el sistema definido por el 3GPP como evolución de LTE (véase Figura 1), que cumple los requisitos del IMT-Advanced, considerándose por ello la verdadera tecnología 4G, puesto que LTE es en verdad perteneciente a una generación 3.9. LTEAdvanced ha sido definido en el Release 10 del 3GPP y está destinado a satisfacer los diversos requisitos de las aplicaciones avanzadas que sean comunes en el mercado inalámbrico en el futuro previsible [3].

Hoy en día, antes de emprender el despliegue e implementación de un sistema de comunicaciones móviles, es preciso realizar múltiples simulaciones que permitan al menos de forma aproximada, predecir cómo será el comportamiento de dicho sistema. Esto se debe, entre otros factores, a que un despliegue de semejante magnitud supone gastos considerables para el proveedor de servicio, pues este tipo de sistema concentra su complejidad del lado de la estación base transmisora y simplifica el nivel de usuario.

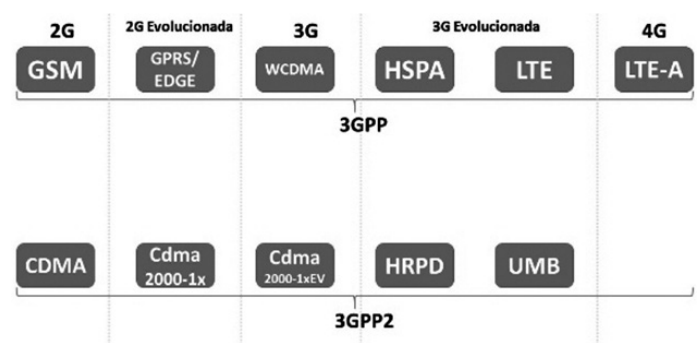

Figura 1. Evolución de los sistemas móviles.

En este sentido, múltiples grupos de investigación se han dedicado al desarrollo de simuladores con la capacidad de emular el comportamiento de dicho sistema. Ejemplo de ello son los simuladores ATOLL y Simu-LTE desarrollados por Forsk y OMNet++, respectivamente [4-5].

Sin embargo, los antes mencionados son softwares propietario, mientras que el LTE Downlink Link Level Simulator, que es el simulador que se ha utilizado en la presente investigación, ha sido desarrollado en la Universidad Tecnológica de Viena y puede ser adquirido gratuitamente. Este simulador es relativamente nuevo (2015) y, por tanto; son escasas las referencias de estudios anteriores realizados con el mismo.

Nuestro trabajo no solo hace un análisis del estándar LTE, sino que también pone a prueba un simulador joven y los resultados obtenidos con él.

En esta investigación se realiza una serie de simulaciones a nivel de enlace donde se evalúa el comportamiento de la tasa de datos del usuario al variar un grupo de parámetros. Se ha estudiado solamente el canal para el enlace descendente de LTE y LTE-Advanced pues es en este sentido en donde se produce el mayor volumen de tráfico habitualmente y, por tanto, en donde se puede hacer una mejor estimación del rendimiento del sistema.

\section{SIMULADORES PARA EL ENLACE DESCENDENTE EN LTE Y LTE-ADVANCED}

Se emplean los simuladores para el enlace descendente de LTE y LTE-Advanced del Instituto de Ingeniería en Comunicaciones y Radiofrecuencia de la Universidad de Viena LTE Downlink Link Level 
Simulator, versión 1.7_r1089 y LTE-Advanced Downlink System Level Simulator, versión 1.3 [6-7].

Estos simuladores están desarrollados sobre MatLab, ofreciendo la facilidad de un código abierto, lo que brinda gran flexibilidad para configurar disímiles entornos, y también permite adaptar el simulador a las necesidades de quien lo usa, pues es posible generar un script propio al estar disponible el código fuente. Tanto las facilidades que ofrece el uso de este tipo de simuladores, como el amplio uso que ya se ha hecho de los mismos en gran cantidad de centros de estudio a nivel mundial, los ha convertido en una selección apropiada para el análisis de los sistemas LTE y LTE-Advanced en la presente investigación.

Estos simuladores son utilizados generalmente a nivel de laboratorio para evaluar elementos teóricos, y así familiarizarse con la arquitectura y el comportamiento de este tipo de sistemas [8].

Aunque permiten simular sistemas LTE y LTEAdvanced, la funcionalidad de este último que ha sido implementada es MIMO mejorado, el que permite emplear hasta ocho antenas en la transmisión y ocho en la recepción [9].

Las simulaciones a nivel de enlace (véase Figura 2) posibilitan la investigación de cuestiones como las técnicas de antena MIMO, la Codificación y Modulación Adaptativa (AMC, Adaptative Modulation and Coding), y el modelo de la capa física a nivel de sistema.

Este simulador permite comprobar el rendimiento de una transmisión LTE o LTE-Advanced (según sea el

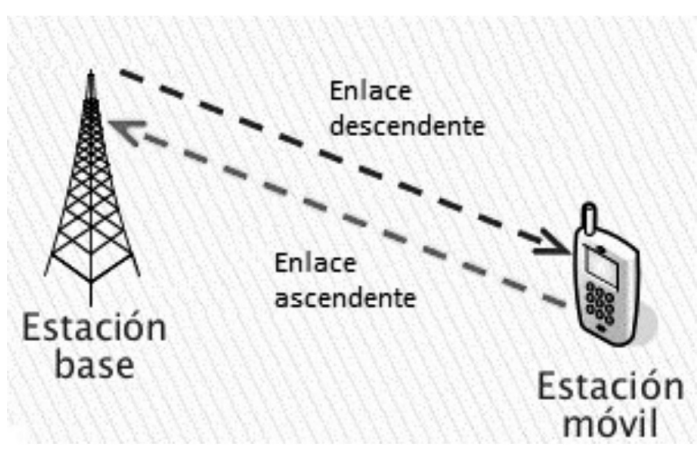

Figura 2. Representación de una simulación a nivel de enlace.
LTE Link Level Simulator o el LTE-Advanced Link Level Simulator) a nivel de enlace, concretamente la tasa media de transferencia de datos exitosa. Es útil para optimizar el diseño de los receptores y analizar los esquemas de modulación y codificación, así como los mecanismos de retransmisión de paquetes, es decir, los diferentes algoritmos de retransmisión, las distintas técnicas y modos de transmisión.

\section{RESULTADOS Y DISCUSIÓN}

\section{Variación de la frecuencia}

En esta simulación se realiza un análisis del desempeño del Throughput [10] de un enlace para valores de $900 \mathrm{MHz}$ y $2.5 \mathrm{GHz}$ de frecuencia. Se analizará el comportamiento de dicha variación de la frecuencia para una serie de modos de transmisión, tanto de LTE (Figura 3) como de LTE-Advanced (Figura 4).

La Tabla 1 muestra los parámetros empleados para realizar la simulación:

Tabla 1. Variación de frecuencia. Parámetros.

\begin{tabular}{|c|c|c|}
\hline \multirow[b]{2}{*}{ Parámetro } & \multicolumn{2}{|c|}{ Valor } \\
\hline & LTE & $\begin{array}{c}\text { LTE- } \\
\text { Advanced }\end{array}$ \\
\hline Número de subtramas & 5.000 & 3.000 \\
\hline Tipo de canal & TU & TU \\
\hline CQI & 7 & 7 \\
\hline $\begin{array}{l}\text { Retransmisiones } \\
\text { HARQ }\end{array}$ & 3 & 3 \\
\hline Ancho de banda & $5 \mathrm{MHz}$ & $5 \mathrm{MHz}$ \\
\hline Modos de transmisión & $\begin{array}{c}\text { SISO, } \\
\text { TxD 2x1, } \\
\text { TxD 4x2, } \\
\text { OLSM 4x2, } \\
\text { CLSM 4x2 }\end{array}$ & $\begin{array}{c}\text { SUMIMO } \\
8 \times 1,8 \times 2 \\
\text { y } 8 x 8, \\
\text { MUMIMO } \\
4 x 1 \\
\end{array}$ \\
\hline Frecuencia & $\begin{array}{c}900 \mathrm{MHz} \\
2.5 \mathrm{GHz}\end{array}$ & $\begin{array}{c}900 \mathrm{MHz} \\
2.5 \mathrm{GHz}\end{array}$ \\
\hline Frecuencia & $\begin{array}{c}900 \mathrm{MHz} \\
2.5 \mathrm{GHz}\end{array}$ & $\begin{array}{c}900 \mathrm{MHz}, \\
2.5 \mathrm{GHz}\end{array}$ \\
\hline
\end{tabular}

El número de subtramas está directamente relacionado con el tiempo de duración de la comunicación entre el usuario y la estación base mediante la relación 1 subtrama $=1 \mathrm{mseg}[10]$. 

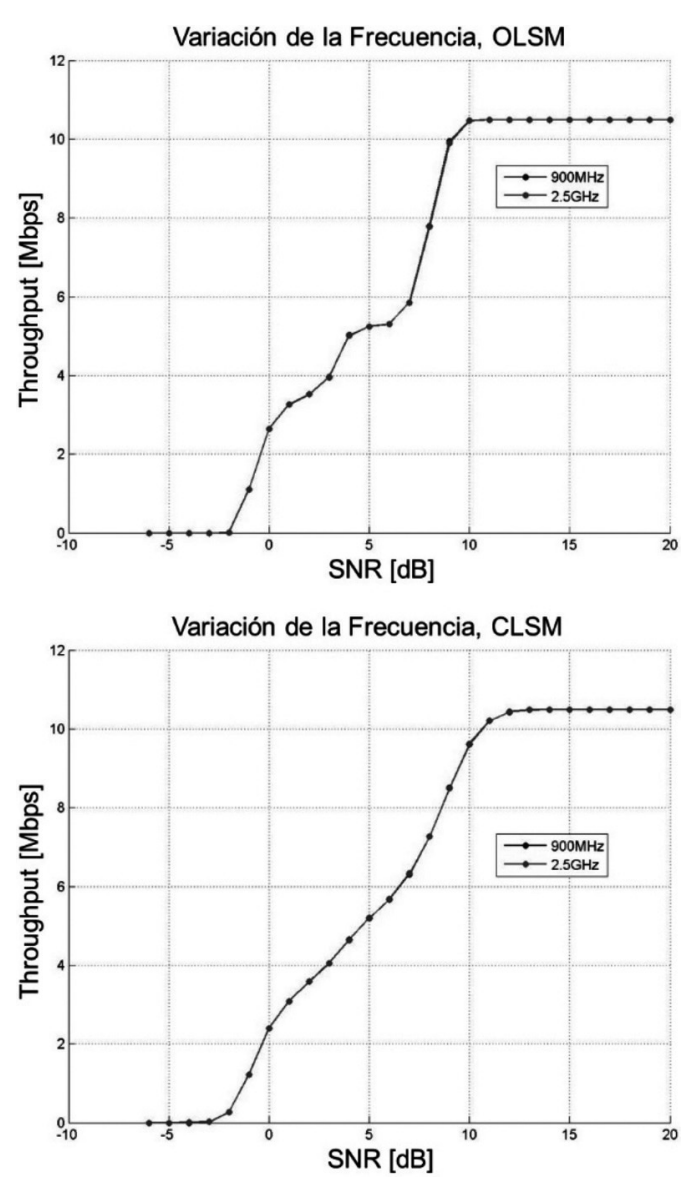

Figura 3. Variación de la frecuencia en LTE. Throughput.

Se ha empleado un canal para simulaciones en un ambiente Típico Urbano (TU). El indicador de calidad del canal (CQI), que puede tomar valores enteros entre 1 y 15 , ha sido fijado en 7 que es un nivel intermedio donde se percibe un nivel aceptable de Calidad de Servicio (QoS) [10].

Ha sido configurado el parámetro de retransmisión del ARQ híbrido en 3, lo que implica que se realizará hasta 3 retransmisiones de un paquete que haya llegado al receptor con un nivel de señal insuficiente.

Los modos de transmisión que han sido configurados, utilizan diferentes técnicas de transmisión, asimismo como configuraciones de antenas en función del estado del canal. Los modos MIMO permiten enviar flujos de información
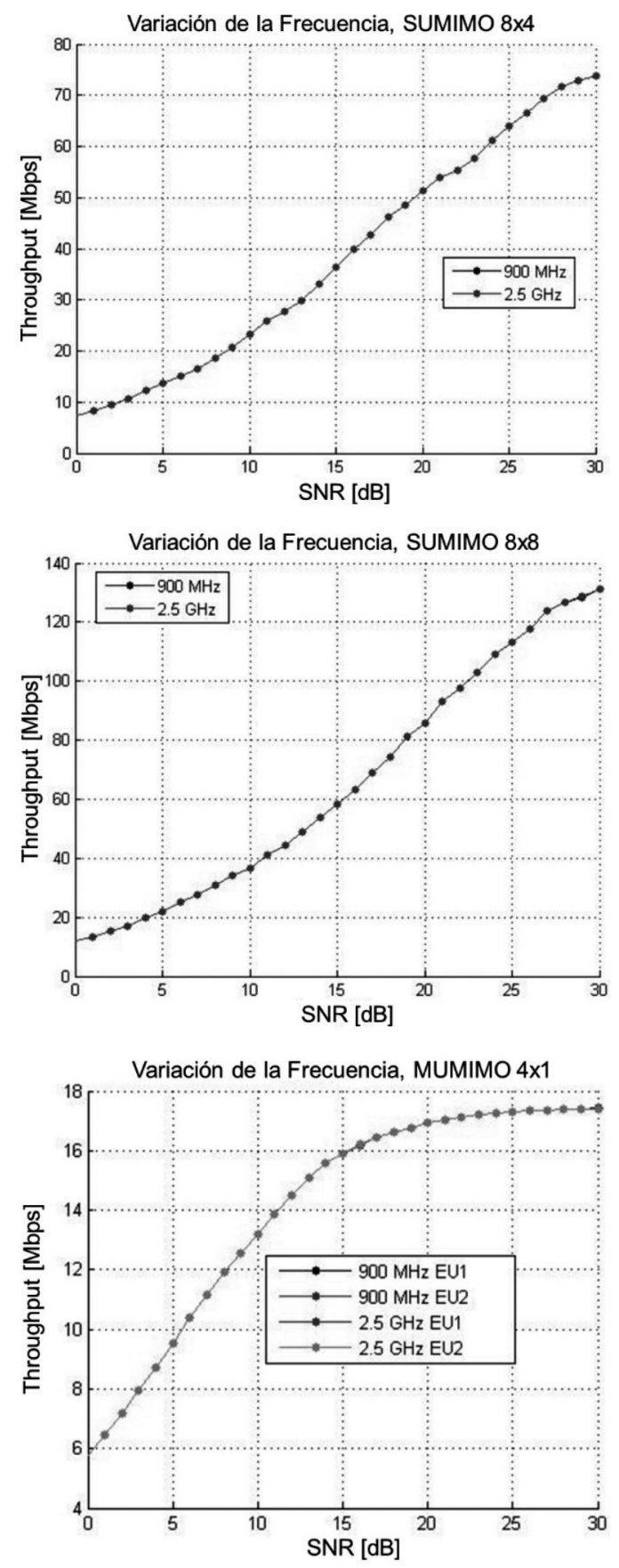

Figura 4. Variación de la frecuencia en LTEAdvanced. Throughput.

diferentes por cada antena, incrementando entonces la capacidad del sistema. Esto es diferente de lo que sucede cuando se emplea un modo de transmisión por diversidad, en donde el mismo 
flujo de información es enviado por múltiples antenas.

OLSM y CLSM (Multilpexación Espacial de Lazo Abierto y Cerrado, respectivamente) son dos modos MIMO de usuario único implementados en el sistema LTE.

\section{LTE}

Las frecuencias empleadas en la simulación son la mínima y la máxima frecuencia permisibles en LTE. Ambas se encuentran dentro de la banda UHF y, por tanto, en ambas se producen fenómenos de propagación similares.

Es posible apreciar que en cada modo de transmisión se obtienen curvas de Throughput similares al producirse una variación en la frecuencia de $900 \mathrm{MHz}$ a $2.5 \mathrm{GHz}$.

\section{LTE-Advanced}

En LTE-Advanced sucede lo mismo que se había observado para LTE. Una variación de la frecuencia, en este caso $900 \mathrm{MHz}$ y $2.5 \mathrm{GHz}$, no se traduce en una variación del Throughput. Al estar ambos sistemas operando en la misma banda de frecuencias, las diferencias que se aprecian en el desempeño de los mismos, no van a estar determinadas por el parámetro frecuencia, sino por otro grupo de parámetros que serán analizados a continuación.

Estas gráficas nos permiten; sin embargo, tener un adelanto de un fenómeno que será analizado con detenimiento en la última simulación. Y es que el modo de transmisión empleado es un parámetro de gran sensibilidad cuando se analiza el comportamiento del Throughput en un canal de radio.

\section{Variación del Ancho de Banda}

Esta simulación realiza un análisis del comportamiento del Throughput de un enlace para valores de 1.4 $\mathrm{MHz}, 3 \mathrm{MHz}, 5 \mathrm{MHz}$ y $10 \mathrm{MHz}$ de ancho de banda. A pesar de que tanto LTE como LTE-Advanced tienen un ancho de banda escalable hasta $20 \mathrm{MHz}$, resulta suficiente para el estudio en cuestión simular hasta $10 \mathrm{MHz}$. Se analizará el comportamiento de dicha variación del ancho de banda para una serie de modos de transmisión, tanto de LTE (Figura 5) como de LTE-Advanced (Figura 6) [10].

La Tabla 2 muestra los parámetros empleados para realizar la simulación:
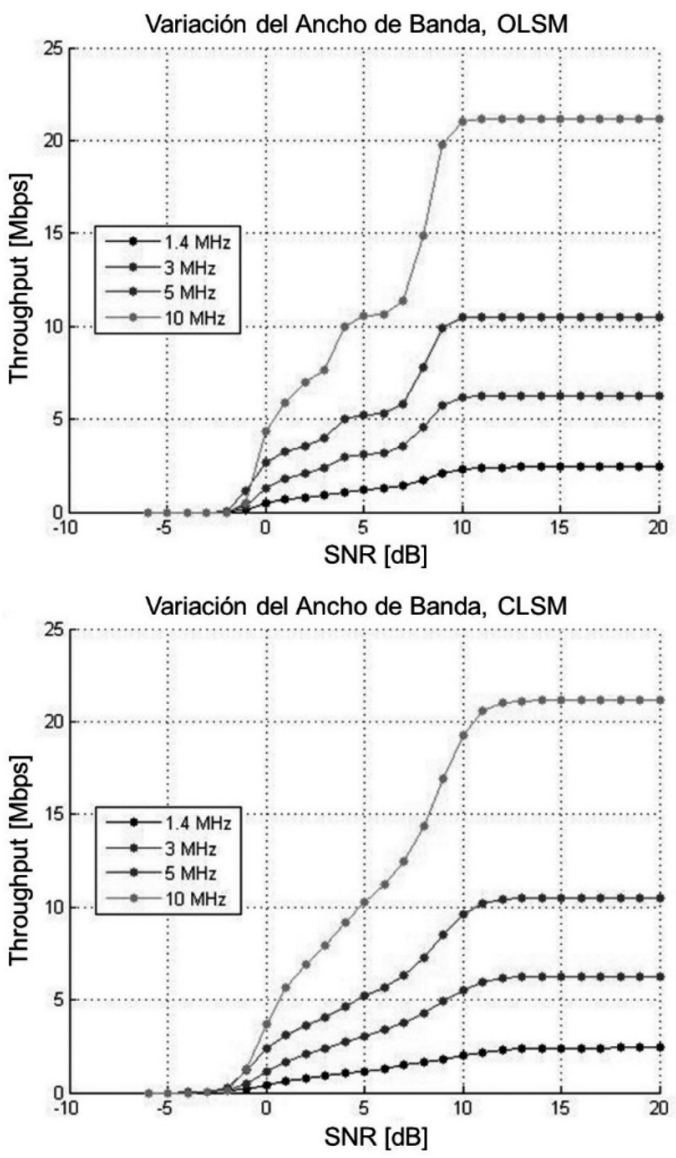

Figura 5. Variación del Ancho de Banda en LTE. Throughput.

Tabla 2. Variación del Ancho de Banda. Parámetros.

\begin{tabular}{|l|c|c|}
\hline \multirow{2}{*}{ Parámetro } & \multicolumn{2}{c|}{ Valor } \\
\cline { 2 - 3 } & LTE & LTE-Advanced \\
\hline Número de subtramas & 5.000 & 3.000 \\
\hline Tipo de canal & $\mathrm{TU}$ & $\mathrm{TU}$ \\
\hline CQI & 7 & 7 \\
\hline $\begin{array}{l}\text { Retransmisiones } \\
\text { HARQ }\end{array}$ & 3 & 3 \\
\hline Frecuencia & $2.5 \mathrm{GHz}$ & $2.5 \mathrm{GHz}$ \\
\hline Modos de transmisión & $\begin{array}{c}\text { SISO, TxD } \\
2 \times 1, \text { TxD 4x2, } \\
\text { OLSM 4x2, } \\
\text { CLSM 4x2 }\end{array}$ & $\begin{array}{c}\text { SUMIMO 8x1, } \\
8 \times 2 \text { y 8x8, } \\
\text { MUMIMO 4x1 }\end{array}$ \\
\hline Ancho de Banda & $\begin{array}{c}\mathbf{1 . 4 M H z ,} \\
\mathbf{3 M H z}, \mathbf{5 M H z}, \\
\mathbf{1 0 M H z}\end{array}$ & $\begin{array}{c}\mathbf{1 . 4 M H z}, \\
\mathbf{3 M H z}, \mathbf{5 M H z}, \\
\mathbf{1 0 M H z}\end{array}$ \\
\hline
\end{tabular}



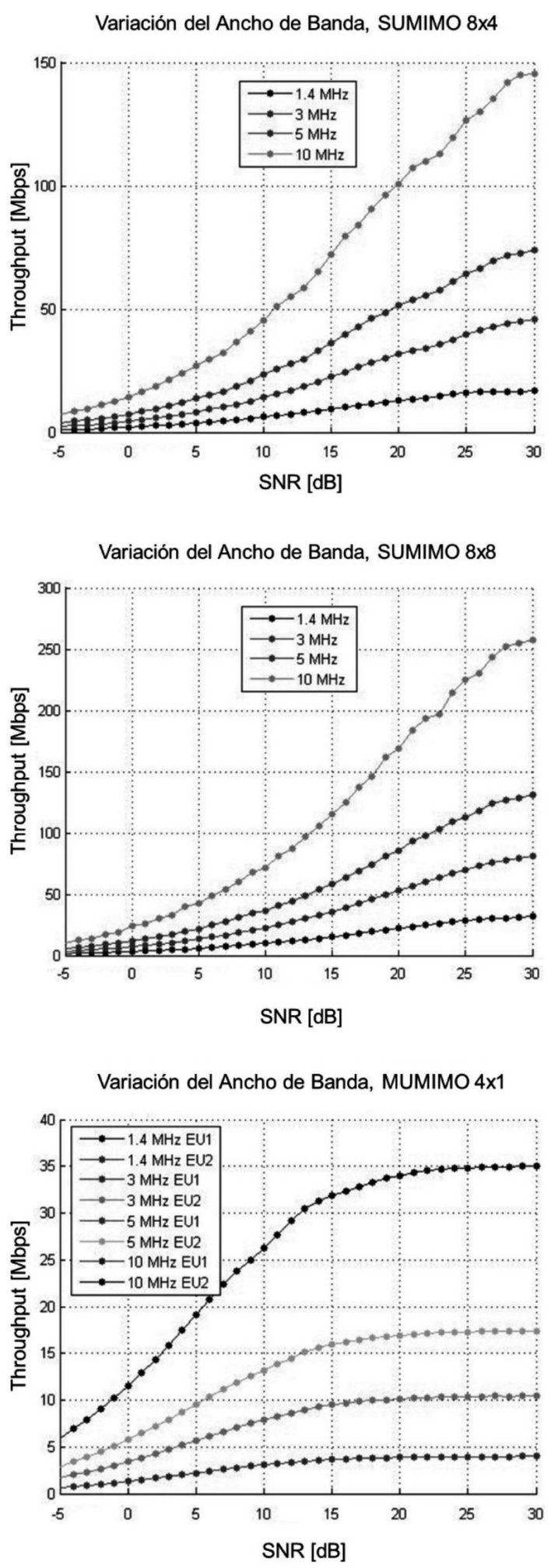

Figura 6. Variación del Ancho de Banda en LTEAdvanced. Throughput.

\section{LTE}

En las gráficas para LTE se aprecia que el Throughput mejora al incrementarse el ancho de banda empleado en cada modo de transmisión.

Es posible observar, ya sea para modo de transmisión OLSM o CLSM que al incrementarse el ancho de banda de $1.4 \mathrm{MHz}$ a $10 \mathrm{MHz}$ se percibe un incremento en el Throughput experimentado por el usuario de cerca de 20Mbps.

Vale destacar que independientemente del ancho de banda empleado los niveles de Throughput se van a estabilizar a partir de los $10 \mathrm{~dB}$ de SNR.

\section{LTE-Advanced}

En LTE-Advanced resulta apreciable que el Throughput experimentado por un equipo de usuario mejora notablemente al incrementarse el Ancho de Banda.

Si se analiza numéricamente este fenómeno es posible apreciar que al incrementarse el ancho de banda de $1.4 \mathrm{MHz}$ a $10 \mathrm{MHz}$, en el modo de transmisión SUMIMO 8x4 se reduce un incremento del Throughput de 130Mbps, en SUMIMO 8x8 de $220 \mathrm{Mbps}$, mientras que para MUMIMO 4x1 este es de $30 \mathrm{Mbps}$. Téngase en cuenta que este último modo de transmisión es para múltiples usuarios a diferencia de los anteriores.

Para ambos sistemas móviles se cumple el mismo principio, y es que el Throughput se incrementa al aumentar el ancho de banda. No obstante es posible apreciar que los resultados obtenidos para LTE-Advanced son mucho mejores que aquellos de LTE.

\section{Variación del tipo de Canal}

En esta simulación se realiza un análisis del comportamiento del Throughput de un enlace para diferentes tipos de canal. Se analizará el comportamiento de cada uno de estos canales para cada modo de transmisión, tanto de LTE (Figura 7) como de LTE-Advanced (Figura 8).

La Tabla 3 muestra los parámetros empleados para realizar la simulación. 

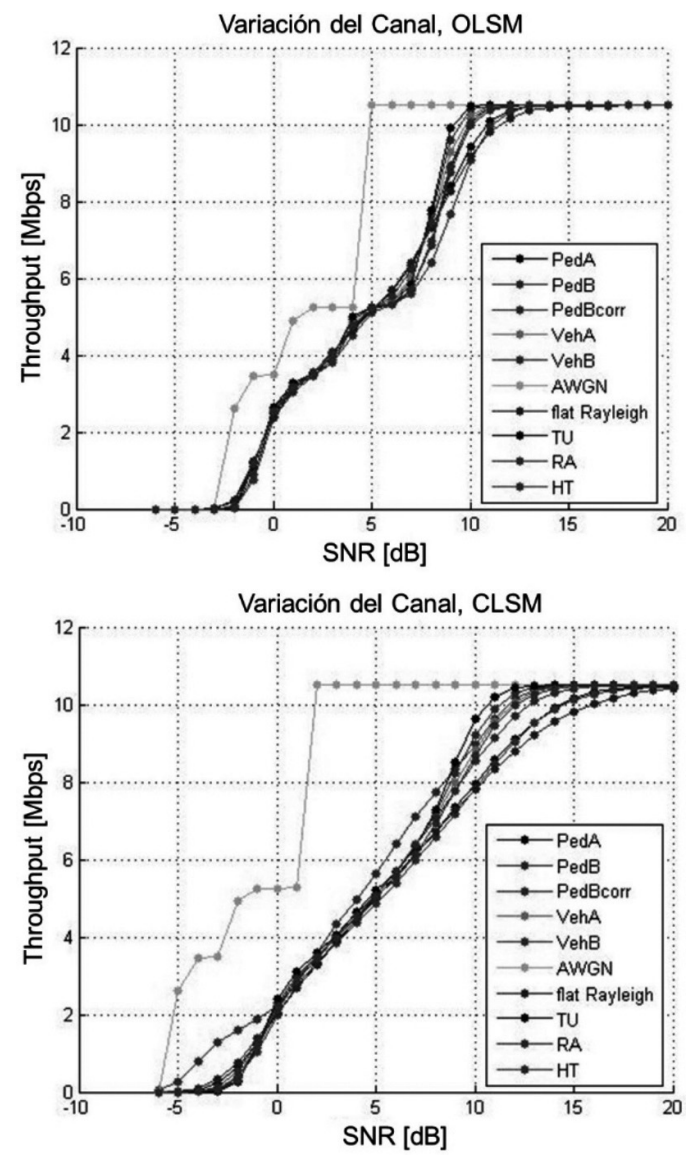

Figura 7. Variación del Tipo de Canal en LTE. Throughput.

Tabla 3. Variación del tipo de Canal. Parámetros.

\begin{tabular}{|l|c|c|}
\hline \multirow{2}{*}{\multicolumn{1}{|c|}{ Parámetro }} & \multicolumn{2}{c|}{ Valor } \\
\cline { 2 - 3 } & LTE & LTE-Advanced \\
\hline Número de subtramas & 5.000 & 3.000 \\
\hline CQI & 7 & 7 \\
\hline $\begin{array}{l}\text { Retransmisiones } \\
\text { HARQ }\end{array}$ & 3 & 3 \\
\hline Frecuencia & $2.5 \mathrm{GHz}$ & $2.5 \mathrm{GHz}$ \\
\hline Ancho de Banda & $5 \mathrm{MHz}$ & $5 \mathrm{MHz}$ \\
\hline Modos de transmisión & $\begin{array}{c}\text { SISO, TxD } \\
2 \times 1, \text { TxD 4x2, } \\
\text { OLSM 4x2, } \\
\text { CLSM 4x2 }\end{array}$ & $\begin{array}{c}\text { SUMIMO 8x1, } \\
\text { MUMIMO 4x1 }\end{array}$ \\
\hline Tipo de Canal & $\begin{array}{c}\text { PedA, PedB, } \\
\text { PedBcorr, } \\
\text { VehA, VehB, } \\
\text { AWGN, flat } \\
\text { Rayleigh, TU, } \\
\text { RA, HT }\end{array}$ & $\begin{array}{c}\text { PedA, PedB, } \\
\text { PedBcorr, } \\
\text { VehA, VehB, } \\
\text { AWGN, flat } \\
\text { Rayleigh, TU, } \\
\text { RA, HT }\end{array}$ \\
\hline
\end{tabular}
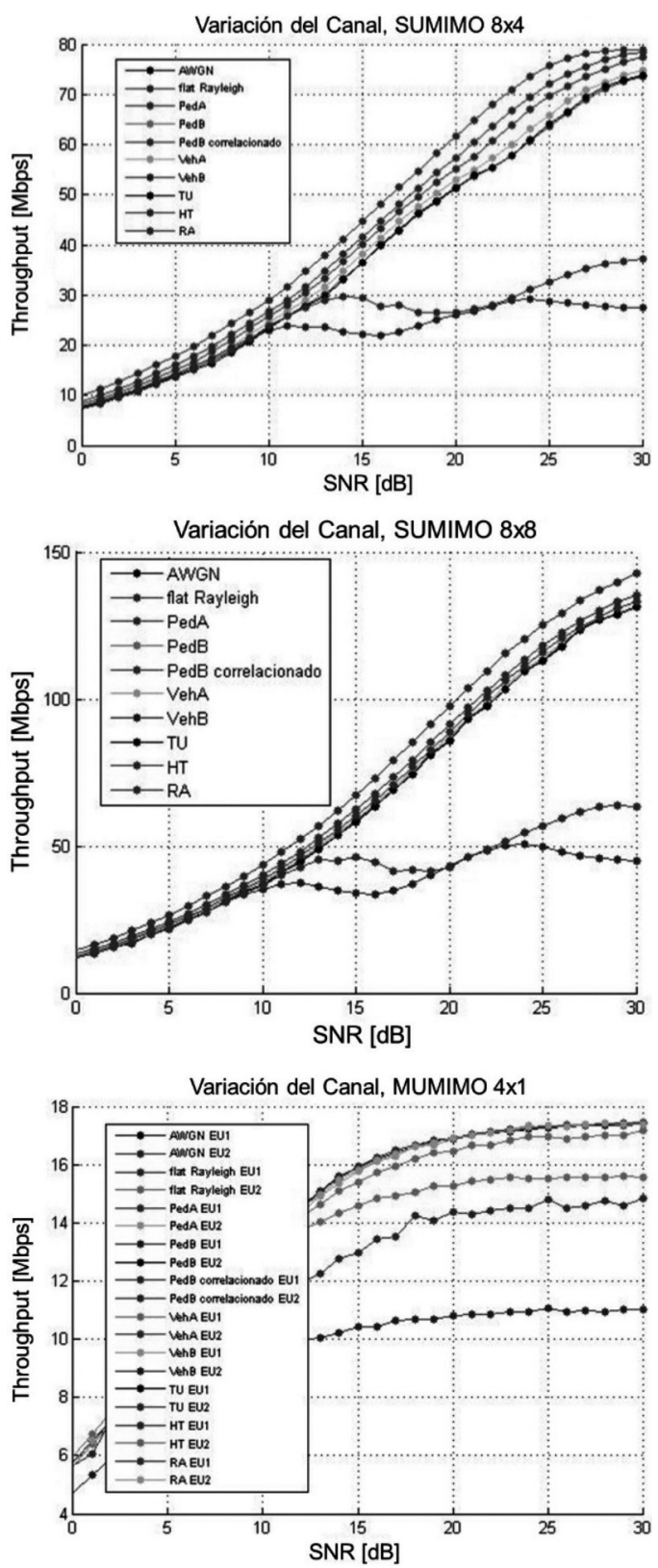

Figura 8. Variación del Tipo de Canal en LTE. Throughput.

Se analizará el comportamiento de los canles peatonales (PedA, PedB y PedBcorr) y vehiculares (VehA y VehB) definidos por la UIT, así como otros de uso más común en el diseño de enlaces de radiocomunicaciones como son un canal Típico Urbano (TU), uno para Área Rural (RA) y uno para Terreno Montañoso (HT). Estos, a su vez, pueden ser comparados con el desempeño ofrecido por 
un canal Rayleigh plano (canal no selectivo en la frecuencia con una distribución Rayleigh) y por uno con Ruido Blanco Aditivo Gaussiano (AWGN).

LTE

En LTE para todos los modos de transmisión el canal que muestra un mejor comportamiento es AWGN, pues consigue estabilizar su valor de Throughput desde un valor de SNR inferior al requerido por los demás canales. Sin embargo, este resultado es de esperar en este tipo de canal ya que no tiene en cuenta una serie de elementos que atenúan la señal en un enlace de comunicaciones móviles como son el multitrayecto, selectividad de la frecuencia, desvanecimientos, interferencias, dispersión, entre otros.

El resto de los canales ofrece un comportamiento similar entre sí para un modo dado, lo que se manifiesta en el comportamiento convergente que se hace evidente en todos los canales, siendo Típico Urbano el canal que logra estabilizar su Throughput para la menor SNR y flat Rayleigh el último en conseguirlo.

El canal que de forma general muestra un mejor desempeño es Típico Urbano.

\section{LTE-Advanced}

En LTE-Advanced, independientemente del modo de transmisión, el canal que muestra mejor comportamiento es flat Rayleigh y el que tiene el comportamiento menos favorable es Vehicular B.

Los canales que exhiben el mejor y el peor desempeño no son los mismos en LTE y en LTE-Advanced. En el caso del primer sistema es un canal Típico Urbano (TU) quien posee el mejor rendimiento, mientras que el que peor lo tiene flat Rayleigh. Sin embargo, en LTEAdvanced son flat Rayleigh y VehB quienes describen el mejor y el peor comportamiento, respectivamente.

Al observar las gráficas de la variación del canal en LTE, es posible apreciar que a pesar de que los canales TU y flat Rayleigh tienen el mejor y el peor comportamiento, respectivamente, existe una convergencia en el comportamiento de todos los canales a partir de cierto valor de SNR. Cuando se hace un análisis semejante para LTE-Advaned, es posible observar que excepto por los canales Vehicular B y Terreno Montañoso, los demás también convergen. Dicha convergencia sugiere que las técnicas de multiplexación espacial MIMO tienen la capacidad de adaptarse a las características de propagación propias de la mayoría de los canales.

El hecho de que en LTE-Advanced flat Rayleigh sea el canal con un mejor desempeño hace pensar que el modo de transmisión MIMO 8x8 se adapta especialmente bien a las condiciones de este canal. Un canal con una distribución de Rayleigh está caracterizado por señales de amplitud similar y fase aleatoria, y este fenómeno es aprovechado por dicha técnica de antenas al utilizar diversidad de polarización para la transmisión [11].

\section{Variación del CQI (Channel Quality Indicator, Indicador de Calidad del Canal)}

Tanto en un sistema LTE como en uno LTEAdvanced, se realiza la misma asignación de CQI (Identificador de Calidad del Canal) dado un valor de SNR determinado. Es posible apreciar en las gráficas de la Figura 9 que a medida que aumenta
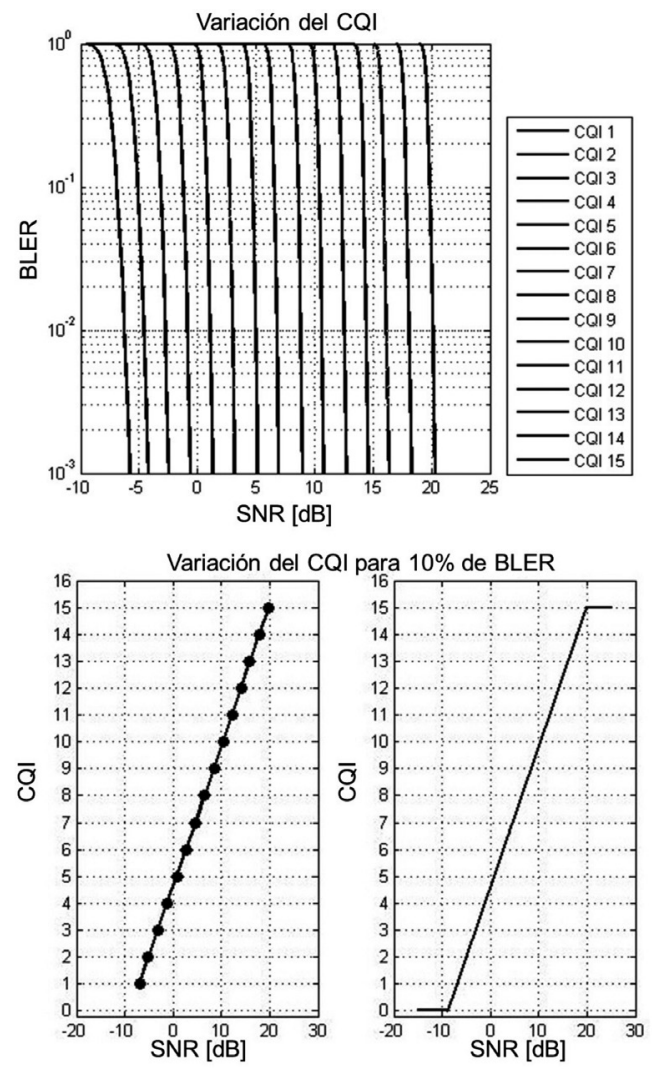

Figura 9. Variación del CQI. BLER (encima) y Valor del CQI (debajo). 
la SNR será asignado al Equipo de Usuario un valor mayor de CQI.

\section{Variación del número de retransmisiones HARQ}

En esta simulación se realiza un análisis del comportamiento del Throughput de un enlace para circunstancias donde se emplean o no retransmisiones HARQ. Se analizará el comportamiento de estos parámetros para una serie de modos de transmisión, tanto de LTE (Figura 10) como de LTE-Advanced (Figura 11), teniendo en cuenta que se emplean 0 (no hay procesos HARQ) y 3 retransmisiones.

La Tabla 4 muestra los parámetros empleados para realizar la simulación:

Tabla 4. Variación del número de retransmisiones HARQ. Parámetros.

\begin{tabular}{|c|c|c|}
\hline \multirow{2}{*}{ Parámetro } & \multicolumn{2}{|c|}{ Valor } \\
\hline & LTE & LTE-Advanced \\
\hline Número de subtramas & 5.000 & 3.000 \\
\hline Tipo de Canal & TU & $\mathrm{TU}$ \\
\hline CQI & 7 & 7 \\
\hline Frecuencia & $2.5 \mathrm{GHz}$ & $2.5 \mathrm{GHz}$ \\
\hline Ancho de Banda & $5 \mathrm{MHz}$ & $5 \mathrm{MHz}$ \\
\hline Modos de transmisión & $\begin{array}{c}\text { SISO, TxD } \\
2 \times 1, \text { TxD 4x2, } \\
\text { OLSM 4x2, } \\
\text { CLSM 4x2 }\end{array}$ & $\begin{array}{l}\text { SUMIMO 8x1, } \\
\text { 8x2 y } 8 \times 8, \\
\text { MUMIMO 4x1 }\end{array}$ \\
\hline Retransmisiones HARQ & 0,3 & $\mathbf{0 , 3}$ \\
\hline
\end{tabular}

\section{LTE}

En todos los modos de transmisión el Throughput se estabiliza en un mismo valor y a partir de una misma SNR, independientemente de la utilización o no de retransmisiones HARQ. Sin embargo, para canales con peores condiciones (valores de SNR inferiores), sí marca una diferencia favorable el empleo de retransmisiones ARQ híbridas.

\section{LTE-Advanced}

Para los modos de transmisión de LTE-Advanced empleados en la simulación, la utilización o no de retransmisiones HARQ no marca una diferencia en el comportamiento del Throughput. Se ha visto que para LTE, el Throughput se mejora al emplearse retransmisiones HARQ; sin embargo, esto no sucede
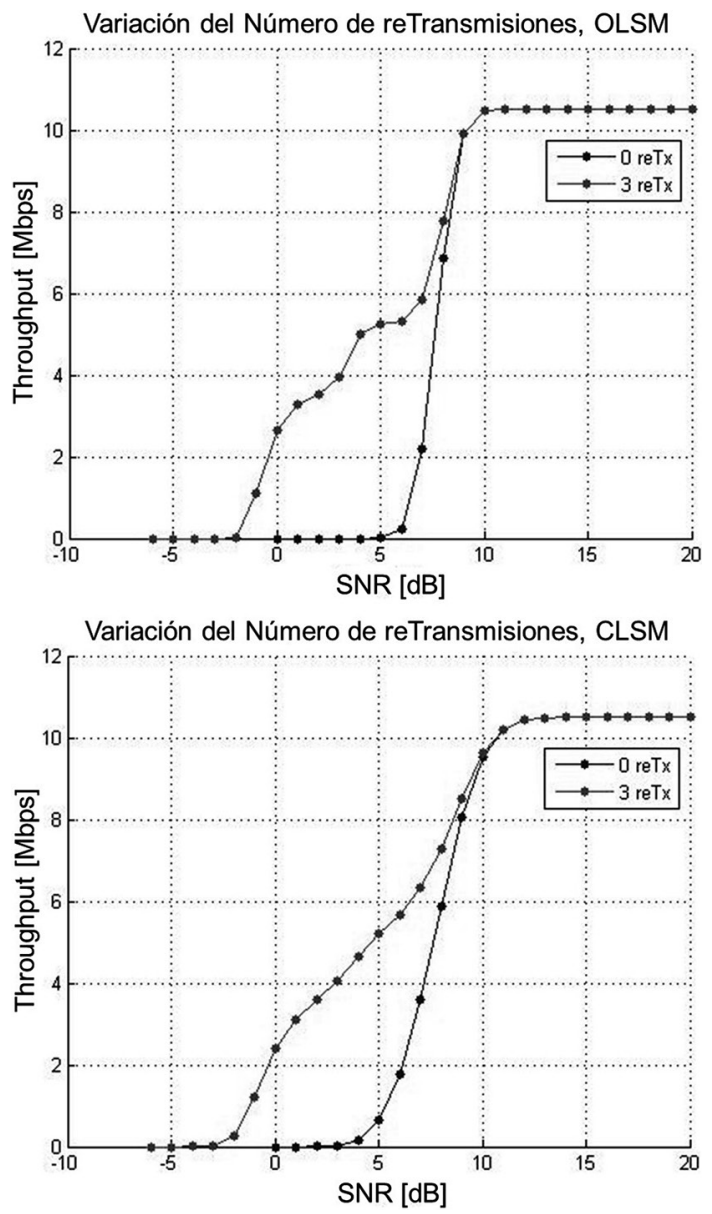

Figura 10. Variación del número de retransmisiones HARQ en LTE. Throughput.

para LTE-Advanced. Los modos de transmisión de LTE-Advanced operan para valores más elevados de la SNR, por tanto, es posible suponer que se produzcan menos errores en la transmisión al aumentar el nivel de señal respecto al ruido del canal y que por tanto el empleo de técnicas ARQ no resulte significativo.

\section{Variación del modo de transmisión}

En cada una de las simulaciones anteriores, a la vez que se ha modificado el parámetro que es objetivo en cada caso se ha ido analizando también el comportamiento de cada uno de los cinco modos de transmisión disponibles para LTE: SISO, TxD 2x1, TxD $4 \times 2$, OLSM $4 x 2$ y CLSM $4 x 2$, y los cuatro que se han empleado en LTE-Advanced: SUMIMO 8x1, SUMIMO 8x4, SUMIMO 8x8 y MUMIMO 4x1. 

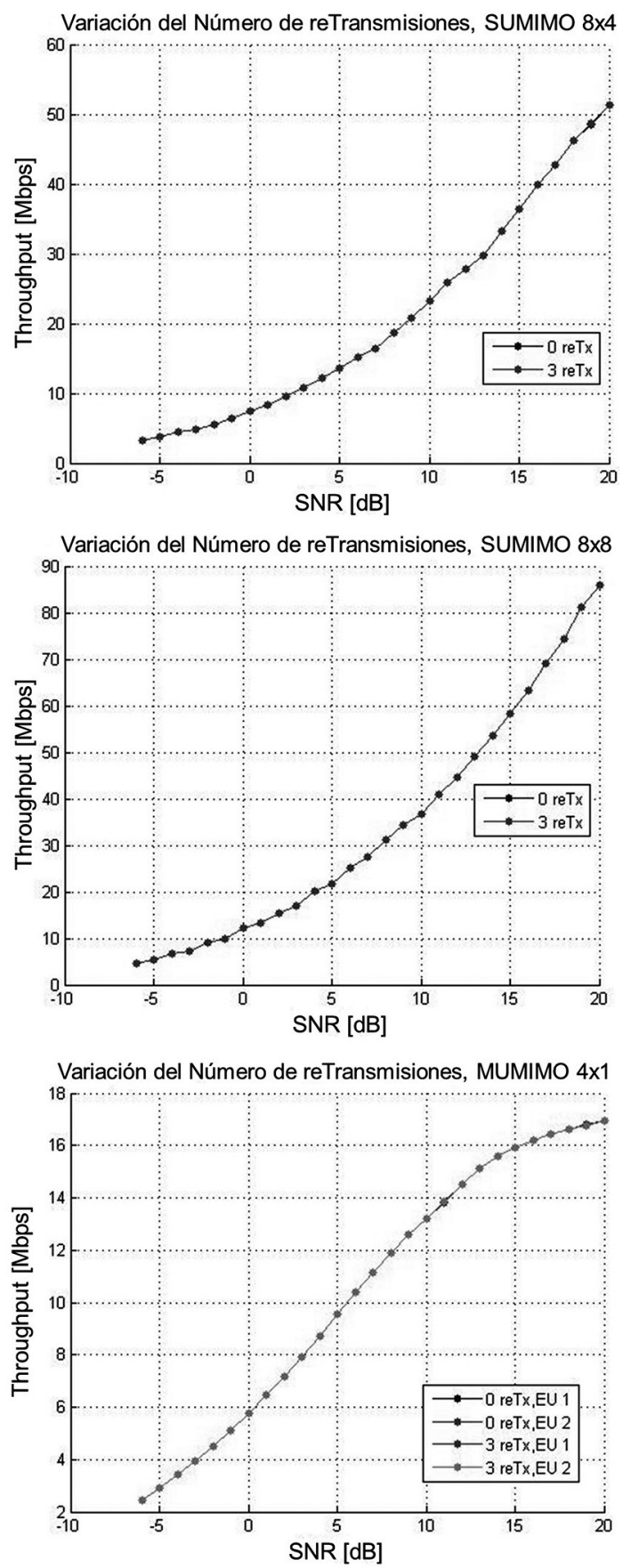

Figura 11. Variación del número de retransmisiones HARQ en LTE-Advanced. Throughput.

Para valorar el comportamiento de cada uno de los modos de transmisión, tanto de LTE como de LTEAdvanced, se muestran dos gráficas en la Figura 12 donde se representa el mejor modo de transmisión de LTE y de LTE-Advanced, respectivamente.
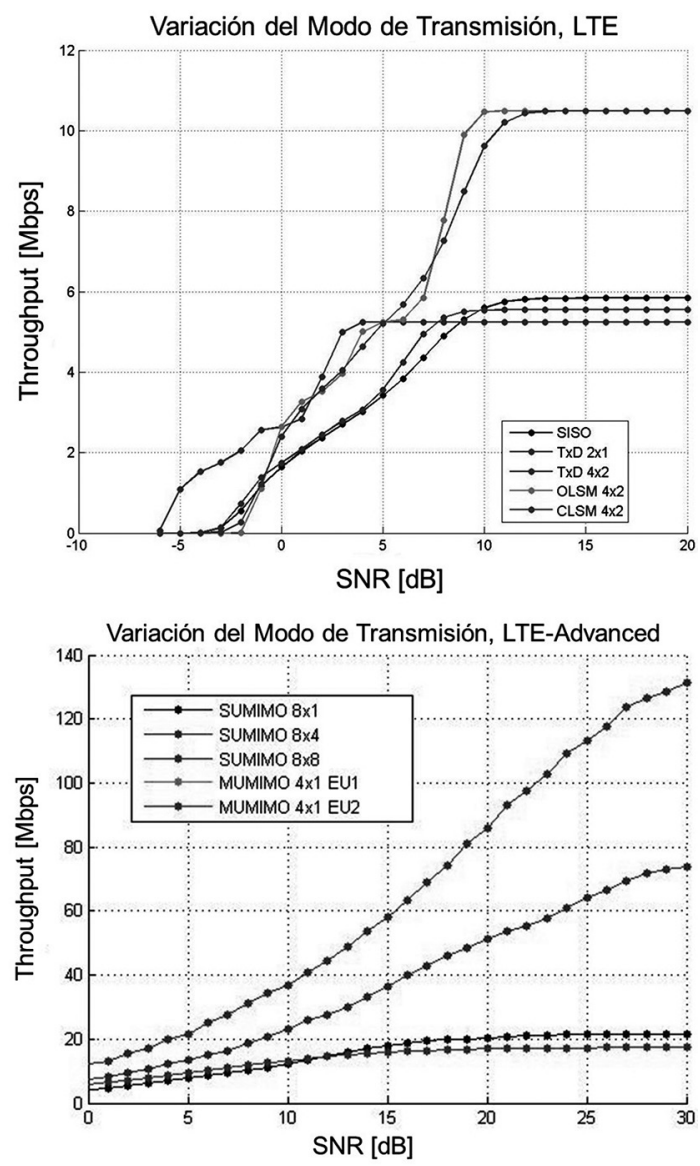

Figura 12. Variación del modo de transmisión, LTE (encima) y LTE-Advanced (debajo)

De las gráficas se puede obsvervar que a medida que aumenta la complejidad del modo de transmisión, aumenta la tasa media de transferencia de datos exitosa (Throughput). Para el sistema LTE, son los modos de transmision OLSM y CLSM los que ofrecen un mejor comportamiento. Es importante destacar que ambos modos se estabilizan para el mismo valor del Throughput lo que se debe a que los dos emplean igual número de niveles en transmsión y recepción.

Mientras, en LTE-Advanced la cantidad de antenas empleadas en transmision y recepcion, asi como el número de usuarios, determina el valor de Throughput alcanzable por usuario.

La Tabla 4 a continuación muestra cómo los modos de LTE que son capaces de alcanzar los valores más altos de Throughput son OLSM y CLSM. 
Asimismo, permite observar que los modos de transmisión de LTE-Advanced, que son una de las mejoras de este estándar (MIMO mejorado) respecto a su predecesor, continúan con la misma tendencia de logar mejores Throughputs que sus antecesores.

Tabla 4. Simulación variación del modo de transmisión. Parámetros.

\begin{tabular}{|l|l|c|c|}
\hline & \multicolumn{1}{|c|}{$\begin{array}{c}\text { Modo de } \\
\text { transmisión }\end{array}$} & $\begin{array}{c}\text { SNR } \\
{[\mathbf{d B}]}\end{array}$ & $\begin{array}{c}\text { Throughput } \\
{[\mathbf{M b p s}]}\end{array}$ \\
\hline LTE & SISO & 12 & 6 \\
\hline \multirow{5}{*}{ LTE-Advanced } & TxD 2x1 & 9 & 5,6 \\
\cline { 2 - 4 } & TxD 4x2 & 4 & 5,2 \\
\cline { 2 - 4 } & OLSM 4x2 & 10 & 10,3 \\
\cline { 2 - 4 } & CLSM 4x2 & 12 & 10,2 \\
\hline \multirow{5}{*}{} & SUMIMO 8x1 & 25 & 21,5 \\
\cline { 2 - 4 } & SUMIMO 8x4 $4 \times 8$ & 30 & 73 \\
\cline { 2 - 4 } & MUMIMO 4x1 & 25 & 130 \\
\hline
\end{tabular}

Se debe hacer notar, sin embargo, que lograr mayores tasas de transmisión está asociado en cada caso a requerimientos cada vez mayores de SNR. Aquí se comprueba que estos modos de transmisión que son capaces de alcanzar altos Throughputs son solo recomendables en entornos con buenos niveles de señal, cuando las circunstancias hacen que los niveles de señal sean más bajos, en lugar de intentar aumentar la potencia de transmisión, lo que se recomienda es utilizar una técnica de diversidad distinta.

\section{CONCLUSIONES}

Conducida esta investigación ha sido posible determinar que los parámetros que son más sensitivos a los cambios son el ancho de banda y el modo de transmisión.

Disponer de más ancho de banda en la celda implica que será percibido un mayor Throughput por el usuario. No obstante, este incremento es limitado, pues el espectro radioeléctrico es un recurso escaso, ya que las bandas de frecuencia donde se está operando en estos momentos son altamente utilizadas por otros servicios, y resulta por tanto necesario diseñar tecnologías que permitan la operación en bandas superiores (SHF y EHF) donde están disponibles mayores anchos de banda.

Los modos de transmisión, que son definidos en función de la cantidad de antenas utilizadas en transmisión y recepción, han probado ser un elemento clave para alcanzar un mayor Throughput; sin embargo, aquellos modos de mayor complejidad son empleados únicamente en circunstancias donde hay buenas condiciones del canal, pues en caso contrario es recomendable emplear técnicas más simplificadas para la transmisión.

Las técnicas de transmisión de mayor complejidad son empleados únicamente en circunstancias donde hay buenas condiciones del canal y, en caso contrario, se acude a técnicas más simplificadas para la transmisión.

Los nuevos modos de transmisión de 8 capas, son la principal mejora que ha aportado este sistema. A expensas de una más elevada SNR, con estos modos de hasta 8 niveles en la transmisión y 8 en la recepción, se pueden alcanzar valores de Throughput que están por encima del doble de aquellos obtenidos con LTE. Sin embargo, a pesar de las tasas de datos superiores alcanzadas con LTE-Advanced, este sistema no ofrecerá su mejor desempeño hasta que no hayan sido desplegadas la mayoría de sus nuevas prestaciones.

Otros de los parámetros simulados como el tipo de canal, el Identificador de Calidad del Canal (CQI), el número de retransmisiones HARQ y el modo de transmisión, son ajustados en función de las características del medio, con el propósito de obtener los mejores beneficios dadas las circunstancias de transmisión.

El desarrollo de nuevos estándares para las comunicaciones móviles responde a la demanda que hoy existe en este sector, y las tecnologías empleadas para formar parte de cada uno de ellos deben ser capaces de aprovechar las características inherentes del medio de transmisión. Se ha encontrado que las técnicas MIMO no solo juegan un papel de gran importancia en los sistemas hoy en funcionamiento, sino que por el momento lo continuarán jugando en futuros estándares. 


\section{REFERENCIAS}

[1] A.H. Khan, M.A. Qadeer, J.A. Ansari and S. Waheed. "4G as a Next Generation Wireless Network". International Conference on Future Computer and Communication. Aligarh, India. 2009.

[2] M. Farooq, M. Ishtiaq Ahmed and U. M Al. "Future Generations of Mobile Communication Networks". Academy of Contemporary Research Journal. Vol. 2, pp. 24-30. 2013. ISSN: 2305-865X.

[3] S. Kumar, "Fourth Generation of Mobile Communication Systems: Evolution, Objectives, Prospects and Challenges". Noviembre 2009.

[4] A. Mohammed Suliman, Y. Alrazig, A. Amin Babiker and M. Nabi. "UMTS vs. LTE Planning by using ATOLL Simulation Tool Case Study: Alazhary City Khartoum State Sudan". International Journal of Engineering. № 24. 01, pp. 4-9. Mayo 2015. ISSN: 2320-6608.

[5] A. Virdis, G. Stea and G. Nardini. "SimuLTE - A Modular System-level Simulator for LTE/LTE-A Networks based on OMNET++". Simulation and Modeling Methodologies, Technologies and Applications (SIMULTECH). Viena, Austria. Agosto 2014.

[6] D. Bosanska, J. Colom Ikuno, G. Lilley, M. Šimko, C. Mehlfürer, S. Pendl, J. Reitterer, M. Rupp, S. Schwarz, Q. Wang and M. Wrulich. "Vienna LTE Simulators Link Level Simulator Documentation, v1.7r1089", pp. 1-11. Octubre, 2011. Febrero, 2015. URL: http://www.nt.tuwien.ac.at/ltesimulator

[7] D. Bosanska, J. Colom Ikuno, G. Lilley, M. Šimko, C. Mehlfürer, S. Pendl, J. Reitterer, M. Rupp, S. Schwarz, Q. Wang, M. Wrulich, M. Meidlinger and M. Müller. "Vienna LTE Simulators LTE-A System Level Simulator Documentation, v1.3", pp. 1-16. Marzo, 2013. Febrero, 2015. URL: http://www.nt.tuwien. ac.at/ltesimulator

[8] H. Taoka, S. Nagata, K. Takeda, Y. Kakishima, $\mathrm{X}$. She, K. Kusume. "MIMO and CoMP in LTE-Advanced", pp. 20-28. Julio 2015. URL: https://www.nttdocomo.co.jp/english/ binary/pdf/corporate/technology/rd/technical_ journal/bn/vol12_2/vol12_2_020en.pdf

[9] L.L. Peterson and B. S. Davis. "Computer networks a systems approach". Morgan
Kaufmann. 5 edición. Vol. 1, pp. 44-48. Burlington, Estados Unidos. ISBN: 978-012-385059. 2011.

[10] R. Agusti Comes, F. Bernardo Álvarez, F. Casadevall Palacio, R. Ferrús Ferre, J. Pérez Romero and O. Sallent Roig. "LTE: Nuevas Tendencias en Comunicaciones Móviles". Fundación Vodafone España. Vol.1, pp. 55-302. Madrid, España. ISBN: 84-934740-4-5. 2010.

[11] J.M. Hernando Rábanos. "Transmisión por Radio". Editorial Centro de Estudios Ramón Areces, S.A. $4^{\mathrm{a}}$ edición. Vol. 1, pp. 101-110. Madrid, España. ISBN: 84-8004-295-8. 2003.

[12] J.M. Hernando Rábanos. "Comunicaciones Móviles". Editorial Centro de Estudios Ramón Areces, S.A. $1^{\text {a }}$ edición. Vol. 1, pp. 101-110. Madrid, España. ISBN: 84-8004-231-1. 2003.

[13] J.M. Huidobro. "Comunicaciones Móviles Sistemas GSM, UMTS y LTE”. Ra-Ma. $1^{\text {a }}$ edición. Vol. 1, pp. 249-310. Madrid, España. ISBN: 978-84-9964-129-4. 2012.

[14] J.F. Ortega Muñoz, H.M. Félix Ibarra, A. García Melchor, G. Galaviz and D.H. Covarrubias Rosales. "Resource assignement without service priorities using cannel quality information in LTE-advanced systems". International Meeting of Electrical Engineering Research ENVIINVIE. Baja California, México. Marzo 2012.

[15] M. Dehghani and K. Arshad. "LTE-Advanced, and the Way Forward". International Symposium on Communications and Information Technologies (ISCIT). Incheon, Korea, 2014.

[16] P. Jun. "Research of Radio Channel Characteristics in Mobile Communication Technology". $7^{\text {th }}$ International Conference on Intelligent Computation Technology and Automation. Changsha, China. 2014.

[17] H. Lee, S. Vahid and K. Moessner. "A Survey of Radio Resource Management for Spectrum Aggregation in LTE-Advanced". IEEE COMMUNICATIONS SURVEYS \& TUTORIALS. 16. 2, pp. 745-760. Mayo 2014. ISSN: 1553-877X. DOI: 10.1109/ SURV.2013.101813.00275.

[18] H. Tan, W. Li, T. Wang, J. Fang and Z. Feng. "Th e Analysis on the Candidate Frequency Bands of Future Mobile Communication Systems". China Communications. 12. Suplemento, pp. 140-149. Diciembre 2015. ISSN: 1673-5447. DOI: 10.1109/ CC.2015.7386162. 